\title{
Single-Phase Velocity Determination Based in Video and Sub-Images Processing: An Optical Flow Method Implemented with Support of a Programmed MatLab Structured Script
}

\author{
Andreas Nascimento1, Edson Da Costa Bortoni², José Luiz Gonçalves², \\ Pedro Antunes Duarte ${ }^{2}$, Mauro Hugo Mathias ${ }^{1}$ \\ ${ }^{1}$ Departamento de Mecânica, Faculdade de Engenharia, Câmpus de Guaratinguetá (FEG), \\ Universidade Estadual Paulista (UNESP), Guaratinguetá, Brazil \\ ${ }^{2}$ Universidade Federal de Itajubá (UNIFEI), Itajubá, Brazil \\ Email: andreas.nascimento@gmail.com
}

Received 12 May 2015; accepted 7 June 2015; published 10 June 2015

Copyright (C) 2015 by authors and Scientific Research Publishing Inc.

This work is licensed under the Creative Commons Attribution-NonCommercial International License (CC

BY-NC).

http://creativecommons.org/licenses/by-nc/4.0/

(c) (i) (8) Open Access

\begin{abstract}
Important in many different sectors of the industry, the determination of stream velocity has become more and more important due to measurements precision necessity, in order to determine the right production rates, determine the volumetric production of undesired fluid, establish automated controls based on these measurements avoiding over-flooding or over-production, guaranteeing accurate predictive maintenance, etc. Difficulties being faced have been the determination of the velocity of specific fluids embedded in some others, for example, determining the gas bubbles stream velocity flowing throughout liquid fluid phase. Although different and already applicable methods have been researched and already implemented within the industry, a nonintrusive automated way of providing those stream velocities has its importance, and may have a huge impact in projects budget. Knowing the importance of its determination, this developed script uses a methodology of breaking-down real-time videos media into frame images, analyzing by pixel correlations possible superposition matches for further gas bubbles stream velocity estimation. In raw sense, the script bases itself in functions and procedures already available in MatLab, which can be used for image processing and treatments, allowing the methodology to be implemented. Its accuracy after the running test was of around 97\% (ninety-seven percent); the raw source code with comments had almost 3000 (three thousand) characters; and the hardware placed for running the code was an Intel Core Duo 2.13 [Ghz] and 2 [Gb] RAM memory capable

How to cite this paper: Nascimento, A., et al. (2015) Single-Phase Velocity Determination Based in Video and Sub-Images Processing: An Optical Flow Method Implemented with Support of a Programmed MatLab Structured Script. Journal of Software Engineering and Applications, 8, 290-294. http://dx.doi.org/10.4236/isea.2015.86029
\end{abstract}


workstation. Even showing good results, it could be stated that just the end point correlations were actually getting to the final solution. So that, making use of self-learning functions or neural network, one could surely enhance the capability of the application to be run in real-time without getting exhaust by iterative loops.

\section{Keywords}

\section{Optical Flow, Single-Phase Velocity, Video and Image Processing, Sensing, MatLab Script}

\section{Introduction}

The industry began to be interested in developing multi-phase-flow-meters, also just called as MPFMs, early in 1980s, since priory, single-phase measurements alone were sufficient to meet the industry's needs. Since 1994, MPFM installation numbers have been steadily increased as technology in the field, with substantial growth witnessed from 1999 onwards [1]. Moreover, in any extension, a solution for detecting single-phase motions embedded in a multi-phase-flow environment may still have its importance and engineering impact.

Studies have shown that non-intrusive flow meters may be more adequate in activities where intrusive ones may affect the effectiveness of the process itself, or where further effort in terms of system re-engineering may show to be necessary, since situations can lead to metering damaging, more frequent maintenance necessity, etc. [2].

Furthermore, since the treatment of the information of this specific idea can be done and run on top of algorithm and media processing, it allows a more reliable real-time data sensing, keeping itself as a very good alternative to be implemented in complex areas or environment where size and intrusiveness of the measuring method may play a bigger role, being these the main motivation of the experiments carried out and detailed in the presented work.

For the developed experiment and simulation, a two-phase flow pattern (clean water and gas bubbles) was used as main fluid combination together with a high resolution recording camera pointing to a transparent tubing. So that non-intrusively, one could record a video from the stream, and subsequently, get the recorded media treated by the developed MatLab script, yielding with the gas bubbles flowing phase velocity determination.

\section{Pixels Correlation Methodology}

Considering that after images treatment one can have in a black-white representative matrix some Booleans specifying what actually represents the effective image and what represents the background, it is possible to have a virtual representation of the gas bubbles in terms of mathematical matrixes.

From the Figure 1 presented, it is possible to see in general what the ideas behind all are. Considering that Figure 1(a) represents one broken-down image from the video media and the Figure 1(b) its subsequent broken-down image, one can understand that the number $01 \mathrm{~s}$ (ones) represent the gas bubbles, and so, by trying to accommodate both on top of each other (each matrix position represents one pixel), it is possible to see that Figure 1(b) advanced in 01 (one) pixel position towards, being its displacement for the final match.

From Figure 2, it is possible to see how the iterative process was developed in order to accommodate the images and the processing basing in pixel correlations on top of each other. Starting from the lowest edge of the matrix, moving column by column from the right to the left and from the downside to the top side, one can see

\begin{tabular}{|l|l|l|l|}
\hline 0 & 0 & 0 & 0 \\
\hline 0 & 0 & 0 & 0 \\
\hline 0 & 0 & 1 & 0 \\
\hline 0 & 0 & 0 & 1 \\
\hline
\end{tabular}

(a)

\begin{tabular}{|l|l|l|l|}
\hline 0 & 0 & 0 & 0 \\
\hline 0 & 1 & 0 & 0 \\
\hline 0 & 0 & 1 & 0 \\
\hline 0 & 0 & 0 & 0 \\
\hline
\end{tabular}

(b)

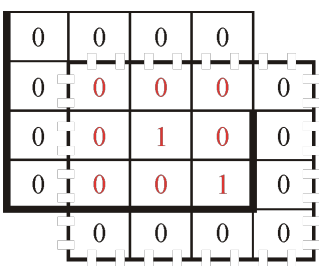

(c)

Figure 1. Main schematic of the correlation matching idea. 


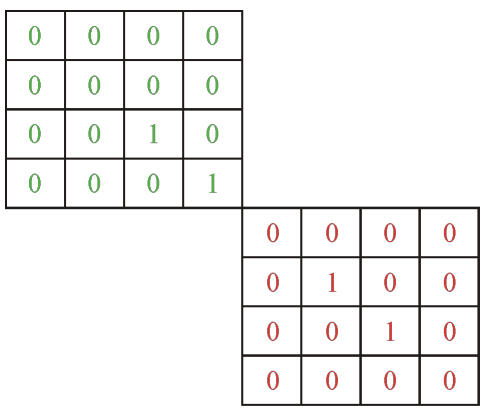

(a)

\begin{tabular}{|l|l|l|l|l|}
\hline 0 & 0 & 0 & 0 & \multirow{2}{*}{} \\
\hline 0 & 0 & 0 & 0 & \multirow{1}{*}{} \\
\hline 0 & 0 & 1 & 0 & \\
\hline 0 & 0,0 & 0,0 & $1<>0$ & 0 \\
\hline \multirow{2}{*}{0} & 0 & 1 & 0 & 0 \\
\cline { 2 - 4 } & 0 & 0 & 1 & 0 \\
\cline { 2 - 4 } & 0 & 0 & 0 & 0 \\
\cline { 2 - 4 } & & & &
\end{tabular}

(d)

\begin{tabular}{|l|l|l|l|l|l|}
\hline 0 & 0 & 0 & 0 & \multicolumn{2}{|c}{} \\
\hline 0 & 0 & 0 & 0 & \multicolumn{2}{|c}{} \\
\hline 0 & 0 & $1<>0$ & 0,0 & 0 & 0 \\
\hline 0 & 0 & 0 & $1=1$ & 0 & 0 \\
\hline \multirow{2}{*nnnnn}{} & 0 & 0 & 1 & 0 \\
\cline { 2 - 4 } & 0 & 0 & 0 & 0 \\
\cline { 2 - 4 } & & & &
\end{tabular}

(g)

\begin{tabular}{|c|c|c|c|c|c|c|}
\hline 0 & 0 & 0 & 0 & & & \\
\hline 0 & 0 & 0 & 0,0 & 0 & 0 & 0 \\
\hline 0 & 0 & 1 & 0,0 & 1 & 0 & 0 \\
\hline 0 & 0 & 0 & $1<>0$ & 0 & 1 & 0 \\
\hline & & & 0 & 0 & 0 & 0 \\
\hline
\end{tabular}

(i)

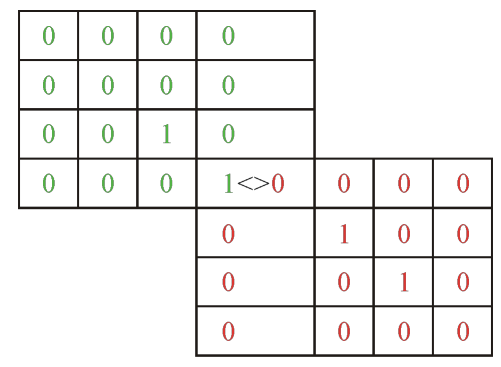

(b)

\begin{tabular}{|l|l|l|l|}
\hline 0 & 0 & 0 & 0 \\
\hline 0 & 0 & 0 & 0 \\
\hline 0 & 0 & 1 & 0 \\
\hline 0,0 & 0,0 & 0,0 & $1<>0$ \\
\hline 0 & 1 & 0 & 0 \\
\hline 0 & 0 & 1 & 0 \\
\hline 0 & 0 & 0 & 0 \\
\hline
\end{tabular}

(e)

\begin{tabular}{|l|l|l|l|l|}
\hline 0 & 0 & 0 & 0 & \multirow{2}{*}{} \\
\hline 0 & 0 & 0 & 0 & \multicolumn{1}{|l}{} \\
\hline 0 & 0,0 & $1<>0$ & 0,0 & 0 \\
\hline 0 & 0,0 & $0<>1$ & $1<>0$ & 0 \\
\hline \multirow{2}{*}{0} & 0 & 0 & 1 & 0 \\
\cline { 2 - 4 } & 0 & 0 & 0 & 0 \\
\cline { 2 - 4 } & & \multicolumn{2}{c}{}
\end{tabular}

(h)

\begin{tabular}{|c|c|c|c|c|c|}
\hline 0 & 0 & 0 & 0 & & \\
\hline 0 & 0 & 0,0 & 0,0 & 0 & 0 \\
\hline 0 & 0 & $1<>0$ & $0<>1$ & 0 & 0 \\
\hline 0 & 0 & 0,0 & $1<>0$ & 1 & 0 \\
\hline & & 0 & 0 & 0 & 0 \\
\hline
\end{tabular}

(k)

\begin{tabular}{|c|c|c|c|c|c|}
\hline 0 & 0 & 0 & 0 & & \\
\hline 0 & 0 & 0 & 0 & & \\
\hline 0 & 0 & 1 & 0 & & \\
\hline 0 & 0 & 0,0 & $1<>0$ & 0 & 0 \\
\hline & & 0 & 1 & 0 & 0 \\
\hline & & 0 & 0 & 1 & 0 \\
\hline & & 0 & 0 & 0 & 0 \\
\hline
\end{tabular}

(c)

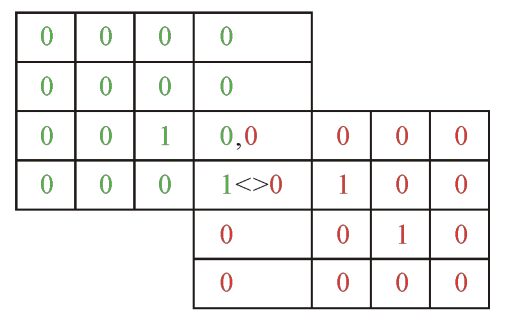

(f)

\begin{tabular}{|l|l|l|l|}
\hline 0 & 0 & 0 & 0 \\
\hline 0 & 0 & 0 & 0 \\
\hline 0,0 & 0,0 & $1<>0$ & 0,0 \\
\hline 0,0 & $0<>1$ & 0,0 & $1<>0$ \\
\hline 0 & 0 & 1 & 0 \\
\hline 0 & 0 & 0 & 0 \\
\hline
\end{tabular}

(i)

\begin{tabular}{|c|c|c|c|c|}
\hline 0 & 0 & 0 & 0 & \\
\hline 0 & 0,0 & 0,0 & 0,0 & 0 \\
\hline 0 & 0,0 & $1=1$ & 0,0 & 0 \\
\hline \multirow[t]{2}{*}{0} & 0,0 & 0,0 & $1=1$ & 0 \\
\hline & 0 & 0 & 0 & 0 \\
\hline
\end{tabular}

(1)

Figure 2. Example of iterative process from beginning up to the processing end match.

the first match appears by Figure 2(g), and the maximum match with the total superposition at the Figure 2(l), which comes to represent the final correlative position for the displacement accountability for further velocity calculation and determination.

Since velocity is not more than a displacement over a specific time-range, putting this information together with the video recording rate in use, one can come-up with the actual effective gas bubbles single-phase stream velocity. Important to notice is that since the background represents the water, for example, it is estimating the total velocity and not the relative velocity on top of the water flow.

\section{MatLab Media Processing Treatments}

The media processing started transforming the images from RGB 3D format (Figure 3(a)) to 2D images, since it has shown to be necessary to have them as singles colors "RGB2GRAY (image)" (Figure 3(b)). 
Sequentially, it was necessary to eliminate isolated pixels in order to allow distinguishing from gas bubbles background using the function "EDGE (image, 'sobel')", and also perform a bordering quality enhancement in order to allow a better delimitation of the bubbles itself (Figure 3(c)).

Finally as a final step, it is always better and necessary to have some pictures boundaries cropped-out, having a better and smoother image to be feeding the script, accomplished using the function IMCROP.

\section{Algorithm Script Schematics Summary Flow-Chart}

From Figure 4 shown, it can be seen how the logic behind the developed algorithm is. The source code itself has been hidden due to specific purposes.

Throughout the analysis and comparison developed with the results, could be verified that this developed methodology and source code script can help as a non-intrusive method of measuring gas bubbles stream velocity in a two-phase or even multi-phase flow environment.

There were two main factors affecting the results that could for sure be enhanced in a next research: better definition of the bubbles sharpness and processing by eliminating the isolated pixels, and guaranteeing to have just perfect ellipses or circles left to be representative so that just raw bubbles would be compared for the pixel superposition matching, allowing a much better accuracy. Furthermore, one can interpret that the matches happens

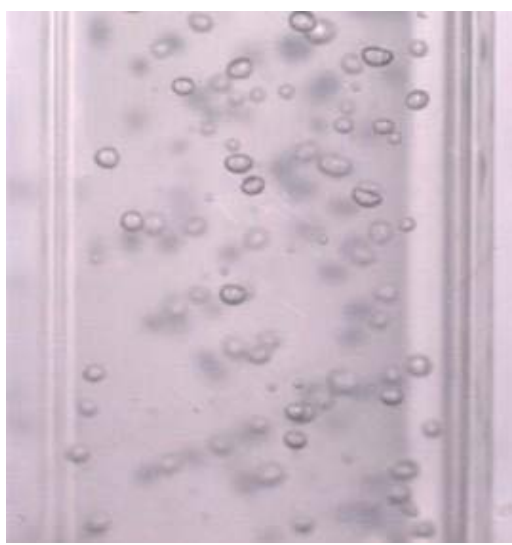

(a)

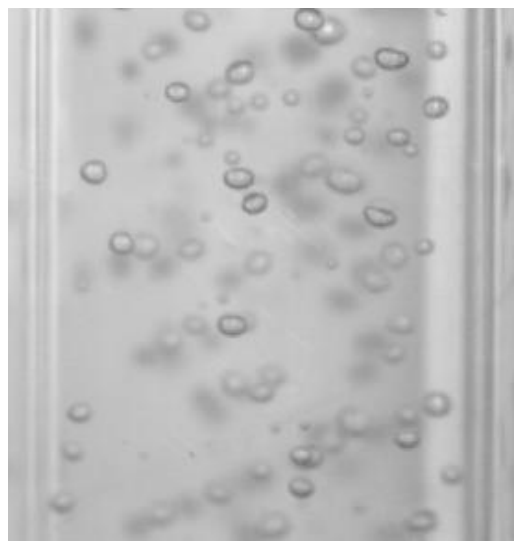

(b)

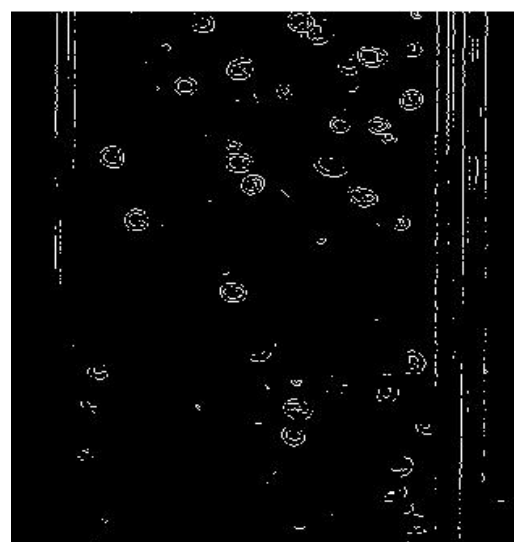

(c)

Figure 3. Media processing from raw 3D format (a) [3] up to final enhanced quality before cropping (c).
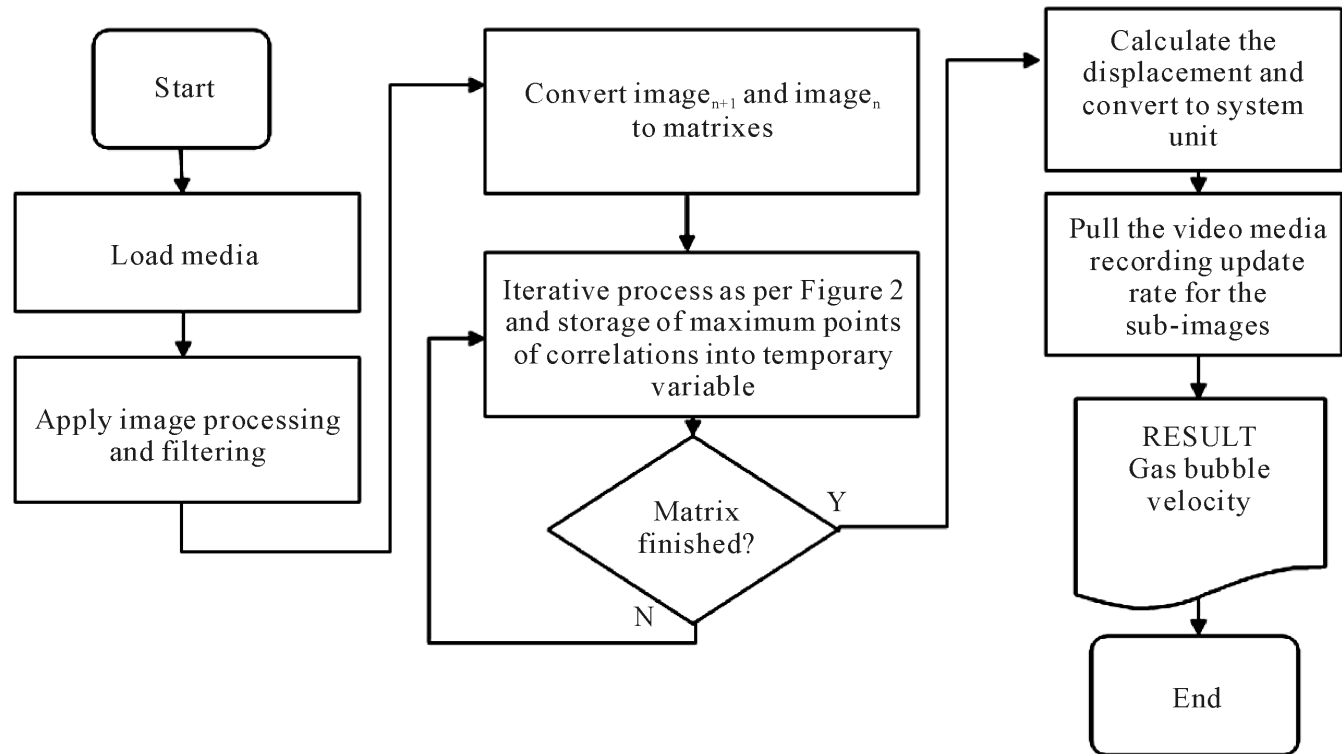

Figure 4. MatLab algorithm flow-chart representation. 
normally on the lasts superposition pixels, so that may not really be necessary to run the correlation code throughout the whole image matrix, but just after a certain specific matrix position. These could lead to source code optimization and results accuracy.

Nevertheless, it allowed an estimation of the stream velocity with an accuracy of around $97 \%$ for the tests run, what is considerable in terms of the necessary work-around implied over these studies and processing.

\section{Conclusions and Discussion}

Throughout the analysis and comparison developed with the results, it could be verified that this developed methodology and source code script can help as a non-intrusive method of measuring gas bubbles stream velocity in a two-phase or even multi-phase flow environment.

There were two main factors affecting the results that could for sure be enhanced in a next research: better definition of the bubbles sharpness and processing by eliminating the isolated pixels, and guaranteeing to have just perfect ellipses or circles left to be representative so that just raw bubbles would be compared for the pixel superposition matching, allowing a much better accuracy. Furthermore, one can interpret that the matches happen normally on the last superposition pixels, so that it may not really be necessary to run the correlation code throughout the whole image matrix, but just after a certain specific matrix position. These could lead to source code optimization and results accuracy.

Nevertheless, it allowed an estimation of the stream velocity with an accuracy of around $97 \%$ for the tests run, what was considerable in terms of the necessary work-around implied over these studies and processing.

\section{Acknowledgements}

This publication has the financial support from the Brazilian Federal Agency for Support and Evaluation of Graduate Education (Coordenação de Aperfeiçoamento de Pessoal de Nível Superior-CAPES, scholarship process no BEX 0506/15-0) and the Brazilian National Agency of Petroleum, Natural Gas and Biofuels (Agência Nacional do Petróleo, Gás Natural e Biocombustíveis-ANP), in cooperation with the Brazilian Financier of Studies and Projects (Financiadora de Estudos e Projetos-FINEP) and the Brazilian Ministry of Science, Technology and Innovation (Ministério da Ciência, Tecnologia e Inovação-MCTI) through the ANP's Human Resources Program of the State University of São Paulo (Universidade Estadual Paulista-UNESP) for the Oil and Gas Sector PRH-ANP/MCTI no 48 (PRH48). Thanks also to the Center of Excellence in Energy Efficiency (Centro de Excelência em Eficiência Energética-EXCEN) from the Federal University of Itajuba (Universidade Federal de Itajubá-UNIFEI).

\section{References}

[1] Mehdizadeh, P. (2006) Worldwide Multiphase and Wet Gas Metering Installations. Production Technology Report 03232007, 2007.

[2] Zuzunaga, A., et al. (2013) A Survey of Non-Invasive and Semi-Invasive Flow Meters for Mining Applications: Understanding and Selecting the Right Technology for the Application. BI0497. International Meeting on Mining Plan Maintenance (MAPLA), Santiago.

[3] Zheng, Y. and Zhang, Q. (2004) Simultaneous Measurement of Gas and Solid Holdups in Multiphase Systems Using Ultrasonic Technique. Chemical Engineering Science, 59, 3505-3514. http://dx.doi.org/10.1016/j.ces.2004.05.016 